\title{
Object Query Optimization through Detecting Independent Sub queries
}

\author{
${ }^{1}$ Mohammad Farhan, ${ }^{2}$ Yatin Chauhan, ${ }^{3}$ Mohammad Akhtar, ${ }^{4}$ Fayeem Khan, \\ ${ }^{5}$ Poonam Pangarkar \\ 1,2,3,4,5 Atharva College Of Engineering,Mumbai, India, 400095.
}

\begin{abstract}
Database Switching includes execution of queries on same or different machines connected in LAN with different backend without rewriting any queries. Switching between the databases reduces the work of rewriting the queries for each of the database thus saving time. User just has to select the Source DBMS and Table from that DBMS and a switch file is created on a click of a button. Now this switch file can be used in any machine (can be a remote machine) to switch data in any of the DBMS. Database GUI helps a user to do all database operations through a GUI provided with minimal knowledge of the database. Here user can do all database activities like Creating Table, Inserting Data, Joining two tables etc with minimal knowledge of queries without having the knowledge of whole database can access through GUI.
\end{abstract}

Keywords: Query Optimization, Query Evaluator, Parser, Query Processor, Optimizers

\section{Introduction}

In some past decades, Relational Database Management System (RDBM) has been established as the technology, handling databases up to terabytes. Relational DBMSs have been extremely successful in the market; however RDBMS lack the mechanisms to deal with complex structured data. Their tabular approach does not allow a suitable modeling of complex hierarchical objects. Most of the applications such as Geographical Information System, CAD, Multimedia, and Engineering etc. are characterized by having to manage complex, highly interrelated information, which was difficult to manage in RDBMS. To combat the limitations of RDBMS and meet the challenge of the increasing rise of the internet and the Web, programmers developed object-oriented databases in 1980.

Querying through GUI to execute complex as well as simple queries has proved to be an efficient system to people who do not have much knowledge about databases.

Switching between the databases reduces the work of rewriting the queries for each of the database thus saving time. The Graphical User Interface generates the queries using Oracle as the database. Similarly other databases can also be used for future work in order to execute the queries using databases such as MySQL, Sqlserver, MS access, etc with the GUI.

\subsection{Database GUI}

This part of the system deals with generating, executing and listening to the queries through speech mode using SAPI software.

\subsection{Database Switching}

User can select among the four databases such as oracle, MySQL, MS Access, SQL server from which the file is to be switched and can switch the file to be executed in any of the four databases.

\section{Overview Of Oodbms}

Minyar Sassi, and Amel Grissa-Touzi proposed the study of "Contribution to the Query Optimization in the Object-Oriented Databases" World Academy of Science, Engineering and Technology1 2005[04].

An OODBMS is the result of combining object oriented programming principles with database management principles. Object oriented includes programming concepts such as encapsulation, polymorphism and inheritance are enforced along with regular database management concepts like Atomicity, Consistency, Isolation and Durability (ACID properties) which lead to system integrity and consistency and support for an ad hoc query language and secondary storage management systems which allow for networks and object programming languages. OODBMS provides much more facilities and flexibility to store more data in database than RDBMS(Relational Database Management System). 


\section{Literature Review}

Michel Bleja proposed the study of Optimization of Object-Oriented Queries Addressing Large and Small Collections of data[02].Query optimization techniques are dependent upon the query model and language. The query model, in turn, is based on the data (or object) model since it defines the access primitives which are used by the query model. These primitives determine the power of the query model. Object-oriented languages allow expressing queries explicitly in the code, which are optimized using the query optimization techniques from the database domain. With respect to this, a formalized object query language (OQL) has been developed that performs optimization of queries at compile time.

J.Plodzien, A.Krake presented the study of Object Query Optimization through Detecting Independent Subqueries to overcome the lacking that were faced in previous studies[01].In recent years, database research has concentrated on object-oriented data models, which allows to store highly structured data. With regard to the data structuring concepts offered, an object-oriented data model can be looked upon as an extension of the nested relational model, which allows to store relations as attribute values.

Managing very large amounts of data, OODB is a system while supporting all the functionality of a relational database system (including queries, transactions, backup and recovery mechanisms), also offers an Object oriented programming language interface, user defined data types, object identifiers and the ability to manage objects persistently. Features that are common in the RDBMS world such as transactions, the ability to handle large amounts of data, indexes, deadlock detection, backup and restoration features and data recovery mechanisms also exist in the OODBMS world.

Database[01] operations typically involve obtaining a database root from the OODBMS which is usually a data structure like a graph, vector, hash table, or set and traversing it to obtain objects to create, update or delete from the database. When a client requests an object from the database, the object is transferred from the database into the application's cache where it can be used either as a transient value that is disconnected from its representation in the database or it can be used as a mirror of the version in the database in that updates to the object are reflected in the database and changes to object in the database require that the object is refetched from the OODBMS.

Query optimization in relational databases is benefited a lot from the simplicity of the data model. This is not the case with the object model. The object-oriented data model is a generalization of the relational one and is believed to eliminate many of its flaws through incorporating modern concepts. Object models are descended of the semantic.

\section{Features Of Oodbms}

A primary feature of an OODBMS is that accessing objects in the database is done in a transparent manner such that interaction with persistent objects is no different from interacting with in-memory objects.

\subsection{Object Oriented Usage Areas:}

Generally, an object database is a good choice when you have the following three factors; business need, high performance, and complex data.

\subsubsection{High Performance}

With complex data, ODBMS will run anywhere from 10 to 1000 times faster than an RDBMS.

\subsubsection{Complex data}

A lack of unique, natural identification.

A large number of many-to-many relationships.

\subsubsection{Business need}

Business objects are base classes, and data storage objects inherit business objects. Client code deals with data storage objects. 


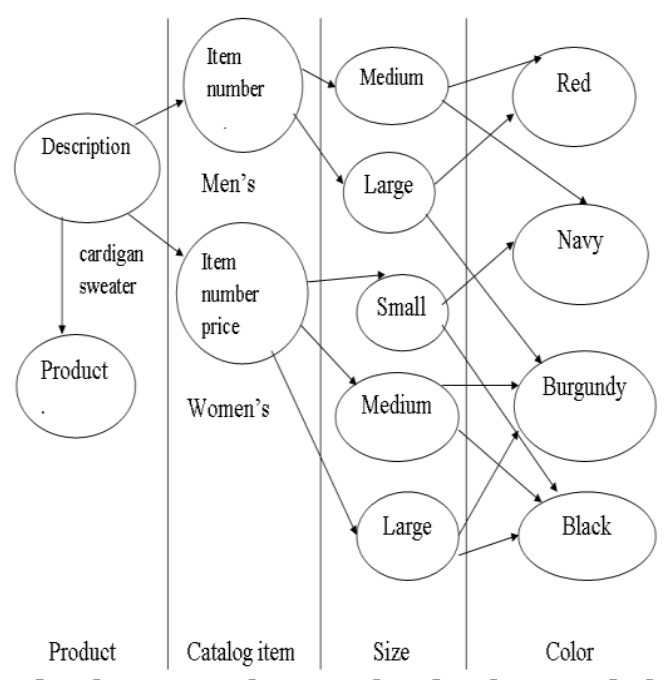

fig 4.1: clothing database uses the complex database and objects.

In the above figure complex database and object is explained in the form of diagram that data is store in database and that to in complex format

\section{Overall Query Processing}

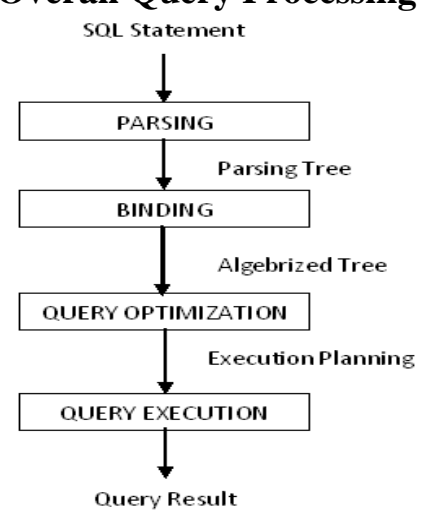

Fig 5.1: The Query Processing Process

Most of the query optimization methods are dependend on query rewriting. Rewriting means transforming a query q1 into semantically equivalent query q2 promising much better performance.

Process of optimization is summarizes in three parts. Parsing of SQL statement is done in face of parse tree, then after parsing binding of statement is carried before the execution plan and finally the execution plan is done and the result of query is displayed.

\section{Architecture}

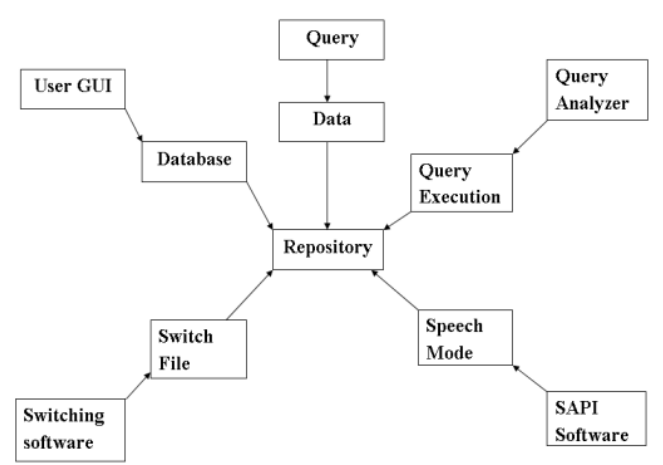

fig 6.1: Architecture of Query Optimization 
User GUI deals with generating and executing queries. User GUI will help the user to work with database query execution and can also learn those queries. User can interact through different GUI components like Drop-down menus, buttons, checkboxes, tool tips etc to learn and execute queries. The GUI part will help the user to avoid the rewriting of queries. SAPI software allows the user with to listen to the queries through speech mode and can correct the queries if entered incorrectly. Switch file will contain the selected records or data that is from the source database and is switched to the selected destination database. Through Query analyser, entered query is analysed before execution to check for semantics and syntax errors.

\section{Comparisons Of Oodbmss To Rdbmss}

"Relational database management implementation is really a process of trying to figure out how to represent real-world objects within the confines of tables in such a way that good performance results and preserving data integrity possibility is high."

"Object database design is quite different. For the most part, object database design is a fundamental part of the overall application design process. The object classes used by the programming language are the classes used by the ODBMS.

\section{Basic Idea Of Proposed Method}

In this paper we are proposing the methods of query optimization. In query optimization, generation of query will be the inter-process.

The idea of this method is based upon the observation that to fetch the input given through GUI.

Interactive input putted by the handler of the GUI will be converted into query through internal process.

\subsection{How the Query Optimizer Works}

At the core of the Oracle Server Database Engine are two major components the Storage Engine and the Query Processor also called the Relational Engine.

Query execution, plan caching: - the query is executed by the Execution Engine, according to the selected plan. The plan may be stored in memory, in the plan cache.

Parsing and binding are the first operations performed when a query is submitted to a ORACLE Server instance. . Parsing makes sure that the ORACLE query has a valid syntax.

The next step is the optimization process

Execution: The DBMS executes the SQL query using the chosen execution plan.

Fetching: The DBMS fetches the data and sends the result sets back to the client.

\section{Conclusion}

In contrast to a traditional setting where users express queries against the database schema, we assert that the semantics of data can often be understood by viewing the data in the context of the user interface (UI) of the software tool used to enter the data. That is, the users will understand the data in a database by seeing the labels, drop-down menus, tool tips, or other help text that are built into the user interface. Database switching allows execution of queries on different machines connected in LAN with different backend without making much effort in writing the query again. Through switching user can save much of the time in rewriting the queries.

\section{References}

[1]. J. Plodzień, A. Kraken, “Object Query Optimization through Detecting Independent Subqueries”, Information Systems, Elsevier Science, 25(8), 2000, pp. 467-490.

[2]. Michel Bleja, Krzysztof Stencel, Kazimierz Subeita, "Optimization of Object-Oriented Queries Addressing Large and Small Collections", Proc. Of the IMCSIT, 2009, ISBN 978-83-60810-22-4, Vol. 4, pp. 643-680.

[3]. K.Subieta, C.Beeri, F.Matthes, J.W.Schmidt. "A Stack-Based Approach to Query Languages". Proc.2nd East-West Database Workshop, 1994, Springer Workshops in Computing, 1995, 159-180.

[4]. Minyar Sassi, and Amel Grissa-Touzi "Contribution to the Query Optimization in the Object-Oriented Databases" World Academy of Science, Engineering and Technology 112005

[5]. G. Gardarin, "Object and relational databases", Eyrolles, 1999

[6]. R.G.G. Catell, "Object-Oriented Data Management: Object-Oriented and Extended Relational Database Sytems”, Addison-Wesley Publishing, Inc., 1994.

[7]. Sunanda Luthra "Architecture In Object Oriented databases"Lecturer, Department of CSE/IT Amritsar College of Engg. \& Tech, Amritsar.143001, Punjab, India .

[8]. David Maier "Object-Oriented Database Theory An Introduction \& Indexing in OODBS" 